\title{
Electroconvulsive Shock Induces Neuron Death in the Mouse Hippocampus: Correlation of Neurodegeneration with Convulsive Activity
}

\author{
I. I. Zarubenko, A. A. Yakovlev, M. Yu. Stepanichev, \\ and N. V. Gulyaeva
}

Translaied from Rossiiskil Fiziologicheskii Zhumal imeni 1. M. Sechenova, Vol. 90, No. 3, pp. 272-281, March, 2004. Original article submitted September 3, 2003.

\begin{abstract}
The relationship between convulsive activity evoked by repeated electric shocks and structural changes in the hippocampus of $\mathrm{Balb} / \mathrm{C}$ mice was studied. Brains were fixed two and seven days after the completion of electric shocks, and sections were stained by the Nissl method and immunohistochemically for apoptotic nuclei (the TUNEL method). In addition, the activity of caspase-3, the key enzyme of apoptosis, was measured in brain areas immediately after completion of electric shocks. The number of neurons decreased significantly in field CA1 and the dentate fascia, but not in hippocampal field CA3. The numbers of cells in CA1 and CA3 were inversely correlated with the intensity of convulsions. Signs of apoptotic neuron death were not seen, while caspase-3 activity was significantly decreased in the hippocampus after electric shocks. These data support the notion that functional changes affect neurons after electric shock and deepen our understanding of this view, providing direct evidence that there are moderate (up to $10 \%$ ) but significant levels of neuron death in defined areas of the hippocampus. Inverse correlations of the numbers of cells with the extent of convulsive activity suggest that the main cause of neuron death is convulsions evoked by electric shocks.
\end{abstract}

KEY WORDS: convulsive activity, electric shocks, hippocampus, apoptosis, caspase-3.

Data on selective cell death in the brain in conditions of convulsive activity were first obtained some 100 years ago [34]. Despite many studies in this area, there is still no unambiguous answer to the fundamental question of whether convulsions are the consequence or cause of cell death in the brain. A number of studies have demonstrated that convulsive activity can develop as a result of brain damage, though there is support for the notion that in some situations, convulsive activity induces brain damage. This latter depends on many factors, primarily the characteristics of the organism (including age), as well as on the type and duration of convulsive activity. For example, status epilepticus induces significant damage in the brain, while repeated convulsive activity in a number of models did not result in cell death [22].

Institute of Higher Nervous Activity and Neurophysiology, Russian Academy of Sciences, 5 a Butlerov Street,

117485 Moscow, Russia.
Convulsive activity can induce neuron death in various parts of the brain. From this point of view, the best studied structure is the hippocampus, as this is believed to be selectively sensitive to the damaging effects of convulsions [7, 36,421 . In adult animals, status epilepticus induces neuron death in hippocampal fields $\mathrm{CA} 1$ and $\mathrm{CA} 3$, granule cells in the dentate fascia, and in the hilus. Cell damage is mediated by excess release of neurotransmitters activating NMDA receptor $\mathrm{Ca}^{2+}$ channels and opening of potential-dependent $\mathrm{Ca}^{2+}$ channels, leading to increased $\mathrm{Ca}^{2+}$ influx into the cell. Excess intracellular $\mathrm{Ca}^{2+}$ leads to a cascade of biochemical events (excessive generation of active forms of oxygen, activation of nitric oxide synthase, uncoupling of oxidation and phosphorylation in mitochondria, and activation of lipases, proteases, endonucleases, and other catabolic enzymes), resulting in cell death [23]. This was demonstrated in animal experiments using a variety of models of convulsive activity, including single-episode status epilepticus induced by kainic acid $[2,7,36,39]$ and by repeated 
convulsions in electric shock $[11,42,49]$ or chemical $[1,38]$ kindling. Along with their fundamental relevance, studies of this type are important for the clinical aspects of epilepsy, because clinical observations, neuropsychological investigations, biochemical studies, and tomographic data indicate that repeated convulsions induce neuron damage in the brains of patients [12]. Thus, recently obtained clinical data from MRI and PET investigations have shown that patients with temporal epilepsy have reductions in hippocampal volume and neuron density in the ipsilateral episode focus of the hemisphere. Changes in the hippocampus are thus worsened with increases in the duration of epilepsy $[12,24,31,45]$.

The question of the mechanisms of hippocampal cell death in convulsive activity also remains open, as some authors have described neuron death as a result of apoptosis, others as a result of necrosis $[11,39,42,49]$, On the other hand, the formation of new neurons in the hippocampus (neurogenesis) has been described in conditions of convulsion-evoked cell death $[29,40,41]$, this evidently representing a repair (compensatory) process.

The maximum electric shock model (generalized convulsive activity resulting from the action of electric currents on animals) has a number of features distinguishing it from the models mentioned above. On the one hand, application of single electric shocks in this model is used as an obligatory test for screening of antiepileptic agents [48]. On the other hand, repeated electric shocks in animals provide a complete analog of electroconvulsive therapy, which continues in clinical use for the treatment of patients with a number of mental illnesses, especially drug-resistant depression [16] resulting from schizophrenia and mania $[5,15]$. The procedure consists of a series of electric shocks provoking generalized convulsive seizures. Treatment courses usually last 2.5 weeks. The standard treatment protocol consists of eight electric shock sessions separated by 48-h intervals [5].

Electroconvulsive therapy is regarded as one of the safest approaches to the treatment of mental illnesses and produces no significant structural changes in the brain [6]. Nonetheless, data have been reported that electric shocks lead to long-lasting functional and anatomical changes in the rat brain [18]. In particular, repeated electric shocks induced long-lasting increases in synaptic efficiency in the dentate fascia [44]. Anomalous axon sprouting has also been observed, in granule cells of the dentate fascia, after electric shocks [46]. Some authors $[10,17]$ have reported increases in the amplitude of the population spike, lasting at least three months after the last electric shock treatment. On the other hand, the treatment has been shown to have protective effects. Thus, Masco et al. [30] found that electric shocks prevented apoptotic cell death in the hippocampus after adrenalectomy, while Kondratyev et al. [25] showed that shocks protected against apoptosis induced by convulsions evoked by kainic acid.

The question of whether cell death occurs in electric shocks remains open. Gombos et al. [18] did not see any significant reduction in the number of cells in the hip- pocampus after electric shocks. Nonetheless, activation of neurogenesis in the hippocampus as a result of electric shocks $[29,40]$ may be a compensatory response to neuron death, as described in other situations.

The aims of the present work were to:

1) determine whether neuron death occurs in the hippocampus after convulsive activity evoked by electric shocks using quantitative assessment of the numbers of cells in hippocampal fields;

2) determine, if cell death was observed, whether it occurs via the apoptotic pathway;

3) to determine whether there is a quantitative relationship between cell death in the hippocampus and convulsive activity induced by electric shocks.

\section{METHODS}

Study system and experimental protocol. Studies were performed using 49 female $\mathrm{Balb} / \mathrm{C}$ mice weighing 18-22 g. Animals were kept with a 12-h light cycle (daytime: 8:00-20:00; night-time: $20: 00-8: 00$ ) with free access to water and food. Mice were divided randomly into three control and three experiments groups. Animals of the group for biochemical investigations were decapitated immediately after the last electric shock, and brain tissues were used for measurements of the enzyme caspase- $3(n=12)$. Two groups of mice were used for morphological studies: 1) animals decapitated two days after the last electric shock session $(n=11)$ and 2$)$ animals decapitated seven days after the last electric shock session $(n=11)$. Each control group (one in the biochemical experimental series and two in the morphological series) consisted of five animals.

Electric shocks. As shown above, repeated electric shocks are an analog of electroconvulsive therapy used in clinical practice $[5,15]$. The procedure consists of a series of electric shocks inducing generalized convulsions. Treatment courses usually last 2.5 weeks and consisted of eight sessions of electric shocks with intervals of $48 \mathrm{~h}$ [5]. We used a similar protocol, as close as possible to the clinical protocol, in our experiments in mice, as described by Scott et al. [40]. Experimental groups of animals were subjected to electric shocks every other day from 11:00 to 14:00, with a total of eight treatments. A direct electric current $(35 \mathrm{~mA}, 0.2 \mathrm{sec})$ was applied via transcorneal electrodes. Each stimulation led to tonic-clonic convulsions lasting 8-10 sec. Mice of all control groups were subjected to the handling process, including transport into the experimental room, manual handling, and placing of electrodes, but not to electric shocks. Convulsions induced by electric shocks were assessed in points on the following scale:

1. Clonic convulsions of the forelimbs.

2. Clonic convulsions with loss of posture.

3. Limb tonus (mainly the hindlimbs).

4. Generalized tonus. 
Estimation of caspase-3 activity. Animals for biochemical studies were decapitated and the cerebral cortex, hippocampus, and cerebellum were removed. Tissues were homogenized in extraction medium $(20 \mathrm{mM}$ HEPES $\mathrm{pH} 7.5,10 \mathrm{mM} \mathrm{KCl}, 1.5 \mathrm{mM} \mathrm{MgCl}, 1 \mathrm{mM}$ dithiothreitol, $1 \mathrm{mM}$ phenylmethylsulfonylfluoride, and $0.5 \mathrm{mM}$ EDTA (all reagents from Sigma, USA) supplemented with protease inhibitors (aprotinin, pepstatin, and leupeptin $(10 \mu \mathrm{g} / \mathrm{ml}$ each), from ICN, USA) at a ratio of 1:5 (weight:volume) in a Potter $\mathrm{S}$ homogenizer (Teflon-glass, Federal Republic of Germany) at a speed of $1500 \mathrm{rpm}$. Homogenates were centrifuged at $14000 \mathrm{~g}$ for $30 \mathrm{~min}$ at $4^{\circ} \mathrm{C}$ and supernatants were used for estimation of caspase-3 activity. Caspase- 3 activity was measured by a fluorimetric method as described by Yakovlev et al. [3]. Supernatants (protein concentration $2 \mathrm{mg} / \mathrm{ml}$ ) were incubated for $60 \mathrm{~min}$ at $37^{\circ} \mathrm{C}$ in reaction buffer $(150 \mathrm{mM}$ HEPES pH 7.5, 15\% sucrose, $15 \mathrm{mM}$ dithiothreitol, $0.15 \%$ CHAPS detergent, $1 \mathrm{mM}$ EDTA (all reagents from Sigma, USA) as two parallel samples, one containing $50 \mu \mathrm{M} \mathrm{N}$-acetyl-Asp-Glu-Val-Asn-7-amino-4trifluoromethylcoumarin (a fluorogenic substrate for caspase-3, from Biomol, USA) and the other containing $25 \mu \mathrm{M}$ $\mathrm{N}$-acetyl-Asp-Glu-Val-Asp-7-amino-4-trifluoromethylcoumarin and $5 \mu \mathrm{M} \mathrm{N}$-acetyl-Asp-Glu-Val-Asp-CHO, a caspase-3 inhibitor (both from Biomol, USA). Fluorescence was recorded on a Hitachi F-3000 (Japan) spectrofluorimeter with excitation and emission wavelengths of 400 and $490 \mathrm{~nm}$ respectively. Enzyme activity was calculated from the differences in substrate degradation rates in samples containing and not containing the specific caspase-3 inhibitor. The fluorescence standard was 7-amino-4-trifluoromethylcoumarin (Sigma, USA). Protein concentrations in samples were determined as described by Bradford [9].

Morphological studies. For morphological analysis and immunohistochemical studies, mice were decapitated and brains were removed and placed in AFA fixative solution $(96 \%$ ethanol:39\% formalin:glacial acetic acid at a ratio of 7:2:1 by volume). After $2 \mathrm{~h}$, brains were placed in $70 \%$ aqueous ethanol and were stored until used. Blocks of size $3-5 \mathrm{~mm}$ were cut from tissues, and these were embedded in paraffin. Frontal sections of thickness $7 \mu \mathrm{m}$ were cut and stained by the Nissl method with cresyl violet, and were used for quantitative analyses. Identification of apoptotic cell death in brain sections was performed using a method based on attachment of digoxigenin-labeled dUTP to the $3^{\prime}$-OH terminals of DNA fragments with terminal deoxynucleotidyltransferase (the TUNEL method), Sections were deparaffinated in xylene and hydrated with a series of ethanol solutions of decreasing concentrations. After washing with PBS, sections were treated with a mixture of ethanol and acetone ( $1: 1$ by volume) for $10 \mathrm{~min}$, washed again with PBS, and loaded with proteinase $\mathrm{K}(20 \mathrm{mg} / \mathrm{ml}$ in $25 \mathrm{mM}$ Tris-HCl pH 6.0) for 15 min. Further processing was with an Apoptag kit (Intergen, USA), used in accord with the manufacturer's instructions. After the procedure, sections were stained with $1 \%$ methylene green.

Hippocampal neuron counts were performed on preparations stained by the Nissl method. Four sections were taken from each brain at the level of the dorsal hippocampus, -1.46 to $-2.06 \mathrm{~mm}$ from the bregma, with intervals of $140 \mu \mathrm{m}$ between sections. Sections for analysis were selected at random. Neuron numbers were assessed in hippocampal field CA1 in strips of length $400 \mu \mathrm{m}(\times 400)$, and in field CA3 in rectangles of size $300 \times 400 \mu \mathrm{m}(\times 400)$, and in the dentate fascia in three fields of vision of size $150 \times 110 \mu \mathrm{m}$ $(\times 1000)$, selected randomly. Cell counts were made using images obtained with a Camedia-4000 digital camera (Olympus Optical, USA) fitted to an Olympus CX-41 microscope (Olympus Optical, Japan). Image processing and cell counts were performed using the Image-PRO Plus program. Neuron densities in hippocampal and dentate fascia fields (numbers of neurons per section) for each animal were expressed as the arithmetic mean neuron density for four sections.

Statistical processing and analysis of results were performed on Statistica for Windows. The significance of differences was assessed using the $t$ test for independent variables. Relationships between behavioral, morphological, and biochemical data were assessed by Spearman rank correlation analysis.

\section{RESULTS}

Morphological studies of brain sections from mice of the control and experimental groups showed no altered cells. Thus, we observed no signs of marked neurodegeneration due to electric shocks. Nonetheless, analysis demonstrated significant reductions in the neuron density in hippocampal field $\mathrm{CAl}$ in the brains of animals treated with electric shocks. Comparison of control animals with experimental animals decapitated two days after the last electric shock session demonstrated a small (by $10 \%$ ) but statistically significant $(p<0.02)$ decrease in the number of cells in field CAl (Fig. 1). The number of cells in field CA1 in mice decapitated seven days after the last electric shock was also significantly less than in controls (by $6 \% ; p<0.04$ ) (Fig. 1).

Counts of cell density in hippocampal field CA3 revealed no significant differences between the control and experimental groups of animals $(262.8 \pm 5.3,254.2 \pm 6.0$, and $247.2 \pm 5.8$ cells per section in the control group and mice two and seven days after the last electric shock session respectively).

In the dentate fascia, neuron density two days after electric shocks were no different from control, though there was a significant difference between control animals and animals decapitated seven days after the last electric shock (by $5 \% ; p<0.03$ ) (Fig. 2). 


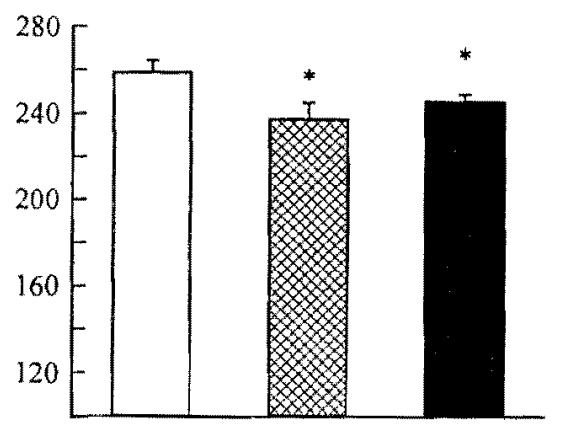

Fig. 1. Effects of electric shock-induced convulsions on the numbers of cells in hippocampal field CAI in mice. The vertical axis shows cell density, neurons/section. Columns show data for groups of animals: the white column shows controls, the shaded column shows experimental animals two days after the last electric shock session, and the black column shows experimental animals seven days after the last electric shock session. $* p<0.05$, Student's $t$ test.

Spearman correlation analysis revealed a significant inverse correlation between the severity of convulsions on the four-point scale and the number of cells in hippocampal field CAl $(r=-0.53 ; p<0.002 ; n=32)$. The inverse correlation between the number of cells in the dentate fascia and the severity of convulsions was less marked and was a tendency $(r=-0.33 ; p<0.07 ; n=32)$.

Immunohistochemical staining for apoptosis did not reveal apoptotic nuclei in the hippocampus of animals of the control or experimental groups.

Measurement of caspase-3 activity in the cerebral cortex revealed no significant difference between experimental and control animals (see Table 1). Comparison of caspase-3 activity in the cerebellum also revealed no significant difference between groups (see Table 1). Caspase-3 activity in the hippocampus of mice subjected to electric shocks was significantly decreased compared with the level of enzyme activity in the control group (by $21 \% ; p<0.005$ ), Caspase-3 activity in the hippocampus of control and experimental animals correlated significantly with the staging of convulsions, such that lower enzyme activity levels corresponded to more marked convulsions $(r=-0.547 ; p<0.03 ; n=17$ ).

\section{DISCUSSION}

This study reports the first quantitative analysis of cells in the hippocampus after convulsions induced by repeated electric shocks. A small (5-10\%) but significant decrease in the number of cells in field $\mathrm{CAl}$ and the dentate fascia was found in experimental animals. Thus, the answer to the first question addressed in this study is positive: neuron death in the hippocampus does occur in electric shocks. Overall, cell death in field CAl was more marked than in the dentate fascia. This may be associated

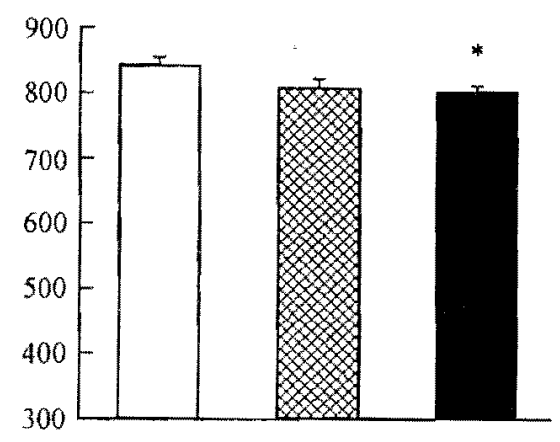

Fig. 2. Effects of electric shock-induced convulsions on cell numbers in the dentate fascia in mice, For further details see caption to Fig. 1

with the different susceptibilities of these hippocampal zones. Another possible explanation of this difference may be the fact that it was in the subgranular zone of the dentate fascia that Scott et al. [40] observed marked neurogenesis after convulsions induced by electric shocks applied using the protocol also used here, Despite the fact that this study did not include quantitative assessment of increases in cells, it can be suggested that the neurogenesis observed was reparative in nature, i.e., the increase in neurogenesis in this situation resulted from death of a proportion of the cells. It is interesting to note that similar results have also been obtained in humans given courses of electroconvulsive therapy: neurogenesis was increased in the dentate fascia of these patients [13]. Thus, cell death may be the stimulus for increased neurogenesis as a compensatory mechanism in a variety of lesions [19]. This partial compensation of the number of cells in the dentate fascia may explain the results obtained in the present study. It should be noted that the small but significant decrease in the number of neurons in the dentate fascia of mice could be detected at seven days (but not at two days) after electric shocks. This may be evidence that unlike the situation in field CAI, neurons in the dentate fascia show progressive continuing death over a longer period after the end of the stimulus. In electric shocks, death may afflict both "old" neurons and neurons appearing in response to convulsive activity-induced neurogenesis but not surviving for various reasons.

The small (in absolute terms) decrease in neuron density may explain the fact that previous morphological analysis did not allow the investigators to detect this phenomenon [18]. Support for the absence of cell death in electric shock in animals, considering data on the absence of neuronal markers in the blood of patients given electroconvulsive therapy [6], also creates the impression that cell death does not occur in convulsions induced by electric 
TABLE 1. Caspase-3 Activity (fmol substrate/min/mg protein) in Brain Areas of Balb/C Mice after Electric Shocks

\begin{tabular}{|c|c|c|c|c|}
\hline \multirow{2}{*}{ Group of mice } & \multirow{2}{*}{$n$} & \multicolumn{3}{|c|}{ Brain area } \\
\hline & & hippocampus & cerebral cortex & cerebellum \\
\hline Control & 5 & $953.8 \pm 89.1$ & $744.0 \pm 90.2$ & $396.2 \pm 42.8$ \\
\hline Electric shock & 12 & $752.9 \pm 21.9 *$ & $817.8 \pm 40.2$ & $447.7=41.6$ \\
\hline
\end{tabular}

Notes. *Significant difference from control, $p<0.005$, Student's $t$ test.

shocks. The detailed quantitative analysis conducted in the present studies demonstrates the converse.

The second question addressed in the present study was associated with the possible involvement of apoptotic neuron death in electric shock-induced neurodegeneration. Despite the formally negative data, the answer to this question may be ambiguous. On the one hand, in fact, it can be suggested that the observed cell death is not associated with apoptosis, as immunohistochemical staining did not demonstrate apoptotic nuclei. Furthermore, rather than the increase in caspase- 3 activity expected in the hippocampus in the presence of apoptosis, we observed a decrease in caspase- 3 activity in the hippocampus of experimental animals. Furthermore, this decrease (by some 20\%) was even more marked than the level of cell death in hippocampal field CA1 and the dentate fascia $(5-10 \%)$. Brain sections stained by the Nissl method showed no necrotic cells or signs of glial activation.

However, despite the absence of evidence supporting apoptotic death, we cannot exclude it. Firstly, it can be suggested that most cell death in field CA1 occurs quite rapidly after the first electric shock sessions. Secondly, if we suggest that cell death in the hippocampus occurs gradually, then our results show that an average of some $0.25-0.5 \%$ of field CA1 cells die per day. Since the total number of cells counted in field CA1 was $240-260$, we were able to see no more than one relevant cell per section in our studies. Similar results were also obtained in the dentate fascia. It should also be noted that apoptotic cells do not remain in the tissue for long periods of time, but are rapidly eliminated by phagocytosis. In other words, our experiments would not be able to demonstrate small numbers of apoptotic cells. Thirdly, despite the prevailing view that activation of caspase-3 is mainly associated with apoptosis of nerve cells, data contradicting this view have also been obtained. Thus, mechanisms of apoptosis without caspase- 3 activation have been observed [14]. Caspase activation in turn does not always lead to apoptosis, but may be involved in the mechanisms of neuroplasticity $[3,4,20,26-28]$. Data obtained by Mattson et al. $[32,33]$ indicate that the enzymes of the apoptotic cascade may alter signaling associated with synaptic plasticity. In particular, it has been established that caspase- 3 can be activated locally in synapses and dendrites without affecting nuclear processes and, consequently, not obligately leading the neuron to apoptosis. Thus, increases in caspase -3 activity are not always evidence of increases in apoptotic processes and, conversely, the presence of apoptosis is not always associated with activation of caspase- 3 . It should also be noted that recent studies have yielded data on the realization of other, caspase-3-independent, pathways of brain neuron death coexisting with apoptotic and necrotic cell death [43]. In view of these points, we can neither confirm nor exclude neuron death by the apoptotic pathway in the electric shock model used here.

The third question addressed in this study related to the possible connection between neuron death and convulsive activity. Despite the fact that the existence of a significant correlation is not evidence for a cause-effect relationship between parameters, the significant inverse correlation between the severity of convulsive activity and neuron density in hippocampal field CA1 suggests that neuron death in this field is a result of convulsions. That fact that this correlation in the dentate fascia was only a tendency may be associated with possible "masking" of such a relationship by increases in neurogenesis in this part of the hippocampus in conditions of electric shocks $([13,44]$, see discussion above). The inverse correlation between the severity of convulsions and caspase- 3 activity would appear to be a direct reflection of cell death due to electric shocks, if we suggest that the mean enzyme activity in cells is not significantly altered by its actions. Our data allow us to provide a positive response to the question of the link between convulsive activity in electric shock and cell death in particular regions of the hippocampus. The experimental conditions (the impossibility of fixing brains for morphological studies and simultaneously measuring caspase -3 within them) prevented analysis of direct correlational relationships between caspase-3 activity and hippocampal neuron death. However, considering the inverse correlations between each of these parameters and convulsive activity, we can suggest that decreases in caspase- 3 activity in conditions of increased convulsive activity are associated primarily with neuron death.

In a recent review [22], Holmes analyzed published data relating to brain damage evoked by convulsive activity and noted that neuron death after repeated convulsions is not as marked as in status epilepticus. This is documented particularly in models of kindling, but has not been demonstrated in conditions of repeated electric shocks [18]. This makes it all the more surprising that a single convulsion can lead to apoptosis of neurons in the brain [8]. Despite the 
absence of support for cell death as a result of electric shocks in previously published reports, many authors have observed long-lived neuronal changes after such shocks. Increases in metabolism were demonstrated [37], along with increases in $f \circ s B$ gene expression, whose target is the gene encoding one of the subunits of the NMDA receptor [21], and long-lasting increases in population spike amplitude in the dentate fascia $[10,44]$. Despite support from several authors for the view that electric shock is not a cause of significant structural damage $[34,37]$ in the brain, longlasting shock-induced sprouting has been demonstrated in the dentate fascia [18].

\section{CONCLUSION}

The data reported here support the concept that significant functional changes following repeated electric shocks do occur; they deepen our understanding of this phenomenon by providing direct evidence for a moderate (up to $10 \%$ ) but significant level of neuron death in particular parts of the hippocampus due to shocks. The inverse correlations between the numbers of cells and the severity of convulsions suggests that convulsions are the main cause of neuron death induced by electric shocks. The approaches used here did not allow us to establish definitively whether neuron death occurs by apoptosis, though they exclude necrotic processes.

This study was supported by the Russian Fund for Basic Research (Grant No. 01-04-49476).

\section{REFERENCES}

1. T. V. Pavlova, A. A. Yakovlev, M. Yu. Stepanichev, A. M. Mendzheritskii, and N. V. Gulyaeva. "Pentylenetetrazolium kindling induces activation of caspase- 3 in the rat brain," $Z$ h. Vysh. Nerv. Deyat., 53, No. 1, 110-112 (2003).

2. M. Yu. Stepanichev, I. M. Zdobnova, I. I. Zarubenko, M. V. Onufriev, Yu. V. Moiseeva, L. 1. Chernyavskaya, and N. V. Gulyaeva, "The intensily of convulsive activity correlates with oxidative stress and apoptosis in the rat hippocampus using the kainate model," Neirokhimiya, 17, No. 3, 189-191 (2000).

3. A. A. Yakovlev, M. V. Onufriev, M. Yu. Stepanichev, K. Braun, and N. V. Gulyaeva, "Caspase- 3 activity in brain areas of various rodent species," Neirokhimiya, 18, No. 1,41-43 (2001).

4. A. A. Yakovlev, T. P. Semenova, S. G. Kolaeva, M. V. Onufriev, S. L. Mikhalev, and N. V. Gulyaeva, "Changes in caspase-3 activity in brain areas of the ground squirrel Citellus undulates during the hibernation cycle," Neirokhimiya, 19, No. 1, 33-36 (2002).

5. R. Abrams, Electroconulsive Therapy, Oxford University Press, New York (1992).

6. M. W. Agelink, J. Andrich, T. Postert, U. Würzinger, T. Zeit, P. Klotz, and H. Przuntek, "Relation between electroconvulsive therapy, cognitive side effects, neuron speciffe enolase, and protein \$-100," J. Neurol, Neurosurg. Psychiat, 71, 394-396 (2001).

7. Y, Ben-Ari, "Limbic seizure and brain damage produced by kainic acid: mechanisms and relevance to human temporal lobe epilepsy," Neurosti, $14,375-403(1985)$.
8. J. Bengzon, Z. Kokaia, E. Elmer, A. Nanobashvili, M. Kokaia, and O. Lindvall, "Apoptosis and proliferation of dentate gyrus neurons after single and intermittent limbic seizures," Proc. Natl Acal. Sci. USA, 94, 10432-10437 (1997).

9. M. M. Bradford, "A rapid and sensitive method for the quantitation of microgram quantities of protein using the principle of protein binding," Anal. Biochem. 72, 248-254 (1976).

10. W. M. Burnham, G. A. Cottrell, D, Diosy, and R. J. Racine, "Longterm changes in entorhinal-dentate evoked potentials induced by electroconvulsive shock seizures in rats," Brain Res., 698, 180-184 (1995).

11. J. E. Cavazos, 1. Das, and T. P. Sutula, "Neuronal loss incluced in limbic pathways by kindling. Evidence for induction of hippocampal sclerosis by repated brief seizures," I. Neuroset, 14, 3106-3121 (1994).

12. J. S. Duncan, "MRI studies. Do seizures damage the brain?" Propt. Bram Res, 135, 253-261 (2002).

13. P. S. Eriksson, E. Perfileva, T. Bjork-Eriksson, A. M. Alborn, C. Nordberg, D. A. Peterson, and F. H. Gage, "Neurogenesis in the adult human hippocampus," Nature.Med., 4, 1313-1317 (1998).

14. B. Fadel, S. Orrenius, and B. Zhivotovksy, "The most unkindest cut of all: on the multiple roles of mammalian caspases," Lezkentia, 14, 1514-1525 (2000).

15. M. Fink, "How does electroconvulsive therapy work?" Neuropharmacol, 3, 73-82 (1990).

16. R. M. Giles, "Electroconvulsive therapy: time to bring it out of the shadows," J. Amer. Med. Assoc, 285, 1346-1348 (2001).

17. Z. Gombos, A. Mentonca, R. J. Racine, G. A. Cottrell, and W. M. Burnham, "Long-term enhancement of entorhimal-dentate evoked potentials following "modified' ECS in the rat," Brain Res., 766, $168-172(1997)$

18. Z. Gombos, A. Spiller, G. A. Cottrell, R. J. Racine, and W. M. Burnham, "Mossy fiber sprouting induced by repeated electroconvulsive shock seizures," Brain Res., 844, 28-33 (1999).

19. E. Gould and P. Tanapat, "Lesion-induced proliferation of neuronal progenitors in the dentate gyrus of adult rat," Neuroset, $80,427-436$ (1997).

20. N. V. Gulyaeva, 1. E. Kudryashov, and 1. V. Kudryashova, "Caspase activity is essential for long-term potentiation," J. Neurosci. Ras.,73, $853-864(2003)$.

21. N. Hiroi, G. J. Marek. J. R. Brown, H. Ye, F. Saudou, V. A. Vaidya, R. S. Duman, M. E. Greenberg, and E. J. Nestler. "Essential role of the fos $\mathrm{B}$ gene in molecular, cellular, and behavioral actions of chronic electroconvulsive seizures," J. Neurosci., 18, 6952-6962 (1998).

22. G. L. Holmes, "Seizure-induced neuronal injury" animal data", Neurology, 59, Suppl. 5, S3-\$6 (2002).

23. G. I. Holmes and $Y$. Ben-Ari, "The neurobiology and consequences of epilepsy in the developing brain" Pediatric Res., $49,320-325$ (2001).

24. R. Kalviainen and T. Samenpera, "Do recurrent seizures cause neuronal damage? A series of studies with MRl volumetry in adults with partial epilepsy," Progr. Brain Res., 135, 279 -295 (2002).

25. A. Kondratyev, N. Sahibzada, and K. Gale, "Electroconvulsive shock exposure prevents neuronal apoptosis after kainic acid-evoked status epilepticus," Brtin Res. Mol. Brain Res., 91, 1-13 (2001).

26. I. E. Kudryashov, M. V. Onufriev, I. V. Kudryashova, and N. V. Gulyaeva, "Periods of postmatal maturation of hippocampal: synaptic modifications and netronal disconnection," Dev. Brain Res., 132 , $113-120(2001)$

27. I. Kudryashov, A. Yakovlev, I. Kudryashova, and N. Gulyaeva, "Footshock stress alters early postnatal development of electrophysiological responses and caspase-3 activity in rat hippocampus," Neurosci. Lett., 332, $95-98$ (2002). 
28. M. Los, C. Stroh, R. U. Janicke, I. H. Engels, and K. SchulzeOsthoff, "Caspases: more than just killers?" Trends Immunol, 22, 31-34 (2001).

29. T. M. Madsen, A. Treschow, J. Bengzon, T. G. Bolwig, O. Lindvall, and A. Tingstrom, "Increased neurogenesis in a model of electroconvulsive therapy," Biol. Psychiatr., 47, 1043-1049 (2000).

30. D Masco, N. Sahibzada, R. Switzer, and K. Gale, "Electroshock seizures protect against apoptotic hippocampal cell death induced by adrenalectomy, Neurosci, 91, 1315-1319 (1999).

31. G. W. Mathern, P. D. Adelson, L. D. Cahan, and J. P. Leite, "Hippocampal neuron damage in human epilepsy: Meyel's hypothesis revisited," Progr. Brain Res., 135, 237-251 (2002).

32. M. P. Mattson and W. Duan, "Apoptotic' biochemical cascades in synaptic compartments: roles in adaptive plasticity and neurodegenerative disorders," J. Neurosci. Res, 58, 152-166 (1999).

33. M. P. Mattson, J. N. Keller, and J. G. Begley, "Evidence for synaptic apoptosis," Exptl. Neurol, 153, 35-48 (1998).

34. B. S. Meldrum, "Concept of activity-induced cell death in epilepsy: historical and contemporary perspectives," Progr. Brain Res., 135 $3-11(2002)$.

35. B. S. Meldrum, "Neuropathological consequences of chemically and electrically induced seizures," Ann. N.Y. Acad. Sci, 462, 186-193 (1986).

36. J. V. Nadler, B. W. Perry, and C. W. Cotman, "Intraventricular kainic acid preferentially destroys hippocampal pyramidal cells," Nature. 271, 676-677 (1978).

37. J. N. Nobrega, R. Raymond, L. DiStefano, and W. M. Burnham, "Long-term changes in regional brain cytochrome oxidase activity induced by electroconvulsive treatment in rats," Brain Res., 605, 1-8 (1993).

38. W. Pohle, A. Becker, G. Grecksch, A. Juhre, and A. Willenberg, "Piracetam prevents pentylenetetrazol kindling-induced neuronal loss and learning deficits": Seizure, 6, 467-474 (1997).
39. H. Pollard, C, CharriautMarlangue, S. Cantagrel, A. Represea, O. Robain, J. Moreau, and Y. Ben-Ari, "Kainate-induced apoptotic cell death in hippocampal neurons," Neurosci, 63, 7-18 (1994).

40. B. W. Scott, J. M. Wojtowicz, and W. M. Burnham, "Neurogenesis in the dentate gyrus of the rat following electroconvuisive shock seizures," Exptl. Neurol. 165, 231-236 (2000).

41. B. W. Scot, S, W. Wang, W. M. Burnham, U. De Boni, and J. M. Wojtowicz. "Kindling-induced neurogenesis in dentate gyrus of the rat," Neurosci. Lett., 248, 73-76 (1998).

42. R. S. Sloviter, "Decreased hippocampal inhibition and a selective loss of interneurons in experimental epilepsy," Science, 235, 73-76 (1987).

43. S. Sperandio, I. De Belle, S. Castro-Obregon, G. del Rio, and D. E. Bredesen, "Cell death programs in neural development and disease," in: Cerebrovascalar Disease, 22nd Princeton Conference, P. H. Chan (ed.), Cambridge University Press, Cambridge (2002).

44. C. Stewart, K. Jeffery, and 1. Reid, "LTP-like synaptic changes following electroconvulsive stimulation," Neuroreport, 5, 1041-1044 (1994).

45. W. H. Theodore and W. D. Gaillard, "Neuroimaging and the progression of epilepsy." Progr. Brain Res. 135, 305-313 (2002).

46. V. A. Vaidya, I. A. Siuciak, F. Du, and R. S. Suman, "Hippocampal mossy fiber sprouting induced by chronic electroconvulsive seizures," Neurosci. 89, 157-166 (1999).

47. R. D. Weiner. "Does electroconvulsive therapy cause brain damage?" Behav. Brain Sci., 7, 1-53 (1984).

48. H. S. White, "Animal models of epileptogenesis," Neurology, $\mathbf{5 9}$ Suppl, 5, S7-S14 (2002).

49. L. X. Zhang, M. A. Smith, X. L. Li, S. R. Weiss, and R. M. Post, "Apoptosis of hippocampal neurons after amygdala kindled seizures," Brain Res. Mol. Brain Res., 55, 198-208 (1998). 